\title{
Pentraxin 3 as A Cardiovascular Marker in Primary Hyperparathyroidism
}

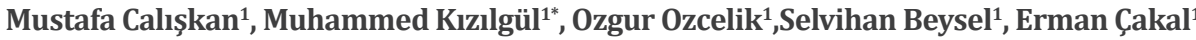 \\ 1Department of Endocrinology and Metabolism, University of Health Sciences, Diskapi Training and Research Hospital, Ankara, Turkey
}

Received: August 03,2018; Accepted: August 16,2018; Published: August 20,2018

*Corresponding author: Muhammed Kizilgul, Department of Endocrinology and Metabolism, Diskapi Training and Research Hospital, Ankara, Turkey,Tel:0905331484820;E-mail: muhammedkzgl@gmail.com

\section{Abstract}

Aim: Pentraxin 3 (PTX3) is an acute-phase glycoprotein, which is increased in patients with cardiovascular disease (CVD) and considered as a predictor of CVD in the general population. Accumulating evidence suggests that even mild primary hyperparathyroidism (PHPT) has an elevated risk for CVD. We aimed to investigate plasma PTX3 levels before and after parathyroidectomy in patients with primary hyperparathyroidism and determine its relationship with cardiovascular risk factors

Material and Method: Twenty-nine patients with PHPT and 26 healthy controls were enrolled in the study. Anthropometric and laboratory parameters were recorded both before and 6 months after parathyroidectomy. Serum PTX3 levels were measured using a human PTX3 enzyme-linked immunosorbent assay.

Results: Plasma PTX3 concentrations were similar between before and after parathyroidectomy and control group $(10.97 \pm$ $16.0,11.97 \pm 11.49,7.88 \pm 9.48$, respectively, $p>0.05$ ). Systolic blood pressure, diastolic blood pressure, carotid intima-media thickness (CIMT) and calcium, parathormone, fasting plasma glucose, alkaline phosphatase concentrations were higher in the PHPT group $(\mathrm{p}<0.05)$. Creatinine and phosphorus concentrations were higher in the control group $(\mathrm{p}<0.05)$. PTX3 was not correlated with cardi-metabolic risk factors except body mass index (BMI) (r2:0.414, p:0.0253).

Conclusion: Plasma PTX3 was not increased as well was not changed after parathyroidectomy in patients with PHPT. The PTX3 was correlated with BMI; however, it was not associated with other cardio-metabolic risk factors including DBP, CIMT, and CRP. These findings might support PTX3 cannot be used as a cardio-metabolic risk marker in patients with PHPT

Keywords: Primary hyperparathyroidism; cardio-metabolic risk factors; pentraxin 3

\section{Introduction}

As an acute-phase glycoprotein, pentraxin 3 (PTX3) has a key role in innate immunity. Additionally, it assists in the removal of degenerated and apoptotic cells. PTX3 plays a role in inflammatory processes, modulates angiogenesis as well as involved in the extracellular matrix formation. Two short pentraxins, C-reactive protein (CRP) and serum amyloid P component, and the long pentraxin, PTX3 are members of the pentraxin family $[1,2]$. PTX3 is synthesized locally in the vascular system by leukocytes, myeloid dendritic cells, fibroblasts, mononuclear macrophages/ phagocytes, and the vascular endothelium in response to stimulation by inflammatory cytokines, however, CRP is mainly produced in the liver upon stimulation by systemic inflammation $[3,4]$. Increased PTX3 concentrations are associated with several disorders including systemic inflammatory response syndrome, sepsis, chronic renal failure, myocardial infarction, cardiac failure, atherosclerosis, vasculitis, lung infections, acute pulmonary damage, lung cancer, eclampsia, rheumatoid arthritis, ankylosing spondylitis and psoriasis [5]. PTX3 concentrations are elevated in heart failure [6], coronary artery disease [7], and acute coronary syndrome [8]. PTX3 levels are increased as well associated with cardiovascular risk factors in both functional and nonfunctional adrenal tumors [9]. Accumulating evidence suggests that even mild PHPT has an elevated risk for CVD [10-14].

We aimed to investigate plasma PTX3 levels before and after parathyroidectomy in patients with primary hyperparathyroidism and determine its relationship with cardiovascular risk factors.

\section{Material and Method}

Twenty-nine patients with PHPT and 26 healthy controls were enrolled in the study. Anthropometric and laboratory parameters were recorded both before and 6 months after parathyroidectomy. Local ethics committee approval from Diskapi Training and Research Hospital was obtained and all participants provided written informed consent before the study began. Patients with multiple endocrine neoplasias, parathyroid cancer, thyroid cancer, hyperparathyroidism-jaw tumor syndrome, and patients on drugs that counteract with calcium and vitamin D metabolism were excluded from the study. The diagnosis of PHPT was defined as persistent hypercalcemia with normal or non-suppressed PTH concentrations [15].

\section{Clinical, Biochemical and Hormonal Measurements}

Basal demographic data, clinical features, carotid intimamedia thickness (CIMT) measurements were recorded for all participants. Weight, height, waist circumference (WC), body mass index (BMI), systolic blood pressure (SDP) and diastolic blood pressure (DBP) were measured. Fasting state biochemical and hormonal measurements were performed in the morning using colorimetric methods and complete blood counts were obtained from all participants. An intact chemilum inescent immunoassay of PTH (Immulite 2000) was used for measurement of serum PTH 
levels. 25-OH vitamin D concentrations were measured using a radio immune assay.

High-resolution B-mode ultrasound (EUB 7000 HV; Hitachi, Tokyo, Japan) with a 13-mhz linear array transducer was used to image parathyroid glands. Carotid intima-media thickness (CIMT) was measured to assess carotid atherosclerosis. CIMT was measured by a B-mode imaging high-resolution ultrasound (EUB 7000 HV; Hitachi, Tokyo, Japan). CIMT is defined as the distance between the blood-intima and media-adventitia boundaries on B-mode imaging high-resolution ultrasound system. Same investigator (MC) performed all ultrasonographic measurements.

\section{Measurement of PTX3}

Serum PTX3 levels were measured using a human PTX3 enzyme-linked immunosorbent assay kit (R\&D Systems Inc, Minneapolis, MN). The sensitivity was $0.116 \mathrm{ng} / \mathrm{mL}$, and the assay range was 0.31 to $20 \mathrm{ng} / \mathrm{mL}$.

\section{Statistical Analysis}

Statistical analysis was performed using JMP 13.0.0 software (SAS Institute, Cary, NC, USA). software. Variables are presented as mean \pm standard deviation (SD) or median, percentage (\%), odds ratio (OR) and $95 \%$ confidence intervals (CI). Normality was tested by Kolmogorov-Smirnov and Shapiro-Wilk W test. Student $\mathrm{t}$ test for normally distributed continuous variables between two groups. Paired sample t-test was used for normally distributed continuous variables before and after the operation. The Chi-square test or Fisher's exact test was used for categorical variables. The correlation was analyzed with the Spearman and Pearson's test. Statistical significance was defined as a $\mathrm{p}<0.05$.

\section{Results}

Mean age, BMI, and gender distribution were similar between groups $(p>0.05)$. Plasma PTX3 concentrations were similar between before and after parathyroidectomy and control group $(10.97 \pm 16.0(\mathrm{ng} / \mathrm{ml}), 11.97 \pm 11.49(\mathrm{ng} / \mathrm{ml}), 7.88 \pm 9.48(\mathrm{ng} /$ $\mathrm{ml}$ ), respectively, $\mathrm{p}>0.05$ ). Systolic blood pressure, diastolic blood pressure, carotid intima-media thickness (CIMT) and calcium, parathormone, fasting plasma glucose, alkaline phosphatase concentrations were higher in the PHPT group $(\mathrm{p}<0.05)$ (Table 1). Systolic blood pressure, diastolic blood pressure and calcium, parathormone concentrations were decreased after parathyroidectomy $(\mathrm{p}<0.05)$. HsCRP, HOMA-IR, total cholesterol, HDL-cholesterol, triglyceride, creatinine and phosphorus concentrations were similar between groups $(\mathrm{p}>0.05)$. PTX3 was not correlated with cardio-metabolic risk factors except body mass index (BMI) (r2:0.414, p:0.0253) (Table 2).

Table 1: Basic demographics of patients and controls

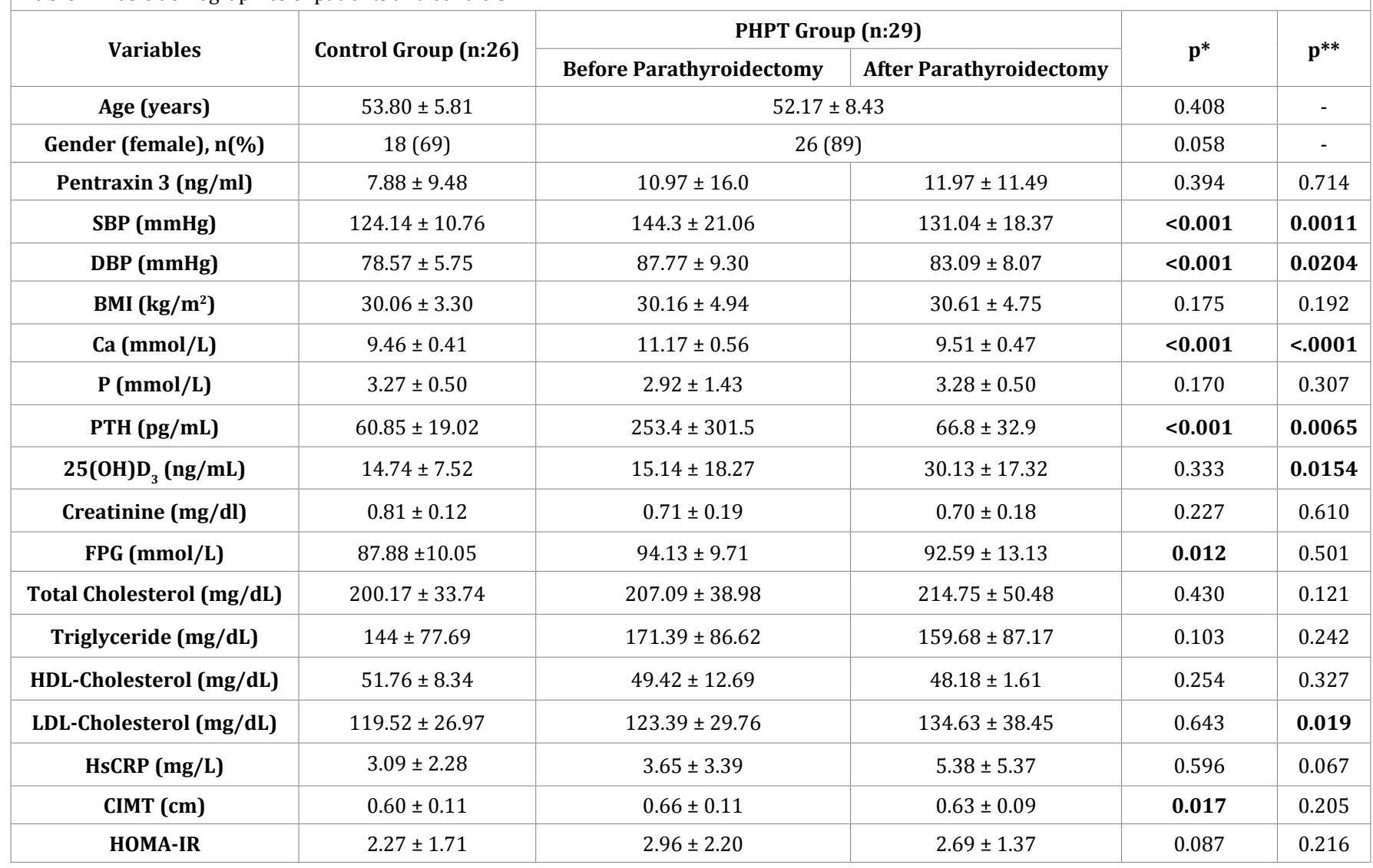

$\mathrm{p}^{*}$ : control group vs. PHPT group preoperatively

$\mathrm{p}^{* *}$ : preoperative PHPT group vs. postoperative PHPT group

SBP: Systolic Blood Pressure; DBP: Diastolic Blood Pressure; BMI; Body Mass Index; PTH: Parathormone; FPG: Fasting Plasma Glucose; CIMT: Carotis Intima Media Thickness 


\begin{tabular}{|c|c|c|}
\hline Table 2: Preoperative correlation analysis of Pentraxin 3 with cardio-metabolic risk factors & p \\
\hline Systolic blood pressure (mmHg) & Correlation Coeficient & 0.188 \\
\hline Diastolic blood pressure (mmHg) & -0.2515 & 0.322 \\
\hline Fasting Plasma Glucose (mg/dL) & -0.1904 & 0.277 \\
\hline LDL-Cholesterol (mg/dL) & -0.2128 & 0.280 \\
\hline Triglyceride (mg/dL) & 0.2114 & 0.324 \\
\hline HDL-cholesterol (mg/dL) & 0.1935 & 0.604 \\
\hline HsCRP & 0.1025 & 0.089 \\
\hline Insulin & 0.3271 & 0.997 \\
\hline HOMA-IR & 0.0008 & 0.818 \\
\hline CIMT (cm) & 0.0485 & 0.695 \\
\hline BMI (kg/m $/ \mathbf{d})$ & 0.0761 & $\mathbf{0 . 0 2 5}$ \\
\hline
\end{tabular}

\section{Discussion}

Our aim was to evaluate the PTX3 concentrations in PHPT patients. Plasma PTX3 was not increased as well was not changed after parathyroidectomy in patients with PHPT. The PTX3 was correlated with BMI; however, it was not associated with other cardio-metabolic risk factors including DBP, CIMT, and CRP. We believe that ours is the first to evaluate the concentrations of PTX3 and its relation to cardio-metabolic risk factors in PHPT.

The association between PTX3 and cardiovascular disorders is well documented [6-8]. The presence of PTX3 in the myocardium and the vasculature with different diseases suggest the elevated concentrations of plasma PTX3 in patients with cardiovascular disorders [16]. The high levels of PTX3 in atherosclerotic plaques and serum of patients with increased LDL cholesterol and widespread atherosclerosis support the potential association between PTX3 and vascular diseases [17-19].

PTH receptors are present in cardiomyocytes, endothelial cells, and smooth muscle cells [20], and patients with myocardial fibrosis, calcification, and hypertrophy have higher PTH concentrations [21]. Many studies have demonstrated PHPT had increased cardiovascular events and mortality which improved after parathyroidectomy [10-14]. Hypertension, hyperlipidemia, CIMT, CRP, and insulin resistance are all well-known risk factors for CVD [22,23]. PHPT patients have higher CVD risk and cardiovascular-related mortality [24]. Several risk factors for CVD have been reported in patients with PHPT, including hypertension, and elevated CIMT, insulin resistance, and CRP [25-28]. In light of this information, we aimed to investigate whether PTX3 could be higher and related to cardio-metabolic risk factors in PHPT patients. However, PTX3 was not higher as well was not changed after parathyroidectomy in patients with PHPT. Additionally, PTX3 concentrations were not correlated with well-known cardio-metabolic risk factors except BMI.

Some studies suggest a positive correlation between PTX3 and BMI $[29,30]$ however, others found a negative correlation $[31,32]$. In our study, we found a positive correlation between
PTX3 levels and BMI.

These findings might be explained by many of our patients possibly being in the early stage of the disease, which might explain why PTX3 was not increased and did not represent an association with all cardio-metabolic risk factors; this is a possible limitation of the study. Additional limitations include the fact that it was a single-center study, and the small sample size was small.

\section{Conclusion}

In conclusion, the PTX3 did not increase in patients with PHPT as well was not changed by parathyroidectomy. PTX3 was correlated with only BMI; however, it was not associated with other cardio-metabolic risk factors. Our findings do not support the thesis of PTX3 as a potential marker of CVD in patients with PHPT. However, more comprehensive studies are necessary to support our findings.

\section{References}

1. Presta M, Camozzi M, Salvatori G, Rusnati M. Role of the soluble pattern recognition receptor PTX3 in vascular biology. Vol. 11, Journal of Cellular and Molecular Medicine. 2007;11(4):723-738. Doi: 10.1111/j.1582-4934.2007.00061.x

2. Bottazzi B. The long pentraxin РTX3 as a link among innate immunity, inflammation, and female fertility. J Leukoc Biol [Internet]. 2006;79(5):909-912. Doi: 10.1189/jlb.1005557

3. CieślikP,HrycekA.Longpentraxin3(PTX3)inthelightofitsstructure, mechanism of action and clinical implications. Autoimmunity. 2012;45(2):119-128. Doi: 10.3109/08916934.2011.611549

4. Deniz T, Kizilgul M, Uzunlulu M, Oguz A, Isbilen B. Levels of Pentraxin 3 and relationship with disease activity in patients with ankylosing spondylitis. Acta Reumatol Port. 2014;39(2):137-142.

5. Kizilgul M, Uzunlulu M, Karacakaya M, Semerci A, Isbilen B IF. Pentraxin 3 Levels as a Marker of Chronic Inflammation in Patients With Metabolic Syndrome. J Endocrinol Metab. 2012;2(6):220-227. 
6. Matsubara J, Sugiyama S, Nozaki T, Sugamura K, Konishi M, Ohba $\mathrm{K}$, et al. Pentraxin 3 is a new inflammatory marker correlated with left ventricular diastolic dysfunction and heart failure with normal ejection fraction. J Am Coll Cardiol. 2011;57(7):861-869. Doi: 10.1016/j.jacc.2010.10.018

7. Guo T, Huang L, Liu C, Shan S, Li Q, Ke L, et al. The clinical value of inflammatory biomarkers in coronary artery disease: PTX3 as a new inflammatory marker. Exp Gerontol. 2017;97:64-67. Doi 10.1016/j.exger.2017.07.018

8. Kume N, Mitsuoka H, Hayashida K, Tanaka M. Pentraxin 3 as a biomarker for acute coronary syndrome: Comparison with biomarkers for cardiac damage. J Cardiol. 2011;58(1):38-45. Doi: 10.1016/j.jjcc.2011.03.006

9. Kizilgul M, Beysel S, Ozcelik O, Kan S, Apaydin M, Caliskan M, et al. PENTRAXIN 3 AS A NEW CARDIOVASCULAR MARKER IN ADRENAL ADENOMAS. Endocr Pract. 2017;23(6):662-668. Doi: 10.4158/EP161713

10. Smith JC, Page MD, John R, Wheeler MH, Cockcroft JR, Scanlon MF, et al. Augmentation of central arterial pressure in mild primary hyperparathyroidism. J Clin Endocrinol Metab. 2000;85(10):35153519. Doi: $10.1210 /$ jcem.85.10.6880

11. Yu N, Donnan PT, Flynn RW V, Murphy MJ, Smith D, Rudman $\mathrm{A}$, et al. Increased mortality and morbidity in mild primary hyperparathyroid patients. the Parathyroid Epidemiology and Audit Research Study (PEARS). Clin Endocrinol (Oxf). 2010;73(1):30-34. Doi: 10.1111/j.1365-2265.2009.03766.x

12. Nilsson IL, Åberg J, Rastad J, Lind L, Clark OH, Perrier ND, et al. Circadian cardiac autonomic nerve dysfunction in primary hyperparathyroidism improves after parathyroidectomy. In: Surgery. 2003;134(6):1013-1039. Doi: 10.1016/j.surg.2003.07.017

13. Piovesan A, Molineri N, Casasso F, Emmolo I, Ugliengo G, Cesario F, et al. Left ventricular hypertrophy in primary hyperparathyroidism. Effects of successful parathyroidectomy. Clin Endocrinol (Oxf). 1999;50(3):321-328.

14. Stefenelli T, Abela C, Frank H, Koller-Strametz J, Globits S, BerglerKlein J, et al. Cardiac abnormalities in patients with primary hyperparathyroidism: implications for follow-up. J Clin Endocrinol Metab. 1997;82(1):106-112. Doi: 10.1210/jcem.82.1.3666

15. Kizilgul M, Caliskan M, Ucan B, Beysel S, Takir M, Sencar E, et al. Monocyte to HDL Cholesterol Ratio and its association with cardiometabolic risk factors in Primary Hyperparathyroidism. Medeni Med J. 2018;33(2):94-99. Doi: :10.5222/MMJ.2018.59837

16. Norata GD, Garlanda C, Catapano AL. The long pentraxin PTX3: A modulator of the immunoinflammatory response in atherosclerosis and cardiovascular diseases. Vol. 20, Trends in Cardiovascular Medicine. 2010;20(2):35-40. Doi: 10.1016/j.tcm.2010.03.005

17. Savchenko AS, Imamura M, Ohashi R, Jiang S, Kawasaki T, Hasegawa G, et al. Expression of pentraxin 3 (PTX3) in human atherosclerotic lesions. J Pathol. 2008;215(1):48-55. Doi: 10.1002/path.2314
18. Bosutti A, Grassi G, Zanetti M, Aleksova A, Zecchin M, Sinagra G, et al. Relation between the plasma levels of LDL-cholesterol and the expression of the early marker of inflammation long pentraxin PTX3 and the stress response gene p66(ShcA) in pacemakerimplanted patients. Clin Exp Med. 2007;7(1):16-23. Doi: 10.1007/ s10238-007-0118-y

19. Ohbayashi H, Miyazawa C, Miyamoto K, Sagara M, Yamashita T, Onda R. Pitavastatin improves plasma pentraxin 3 and arterial stiffness in atherosclerotic patients with hypercholesterolemia. J Atheroscler Thromb. 2009;16(4):490-500.

20. Schluter KD, Piper HM. Cardiovascular actions of parathyroid hormone and parathyroid hormone-related peptide. Cardiovasc Res [Internet]. 1998;37(1):34-41.

21. Fitzpatrick LA, Bilezikian JP, Silverberg SJ. Parathyroid hormone and the cardiovascular system. Vol. 6, Current Osteoporosis Reports. 2008;6(2):77-83.

22. Akhabue E, Thiboutot J, Cheng J-W, Vittorio TJ, Christodoulidis G, Grady KM, et al. New and emerging risk factors for coronary heart disease. Am J Med Sci [Internet]. 2014;347(2):151-158. Doi: 10.1097/MAJ.0b013e31828aab45

23. Cannon CP. Cardiovascular disease and modifiable cardiometabolic risk factors. Clin Cornerstone. 2007;8(3):11-28.

24. Andersson P, Rydberg E, Willenheimer R. Primary hyperparathyroidism and heart disease--a review. Eur Hear J [Internet].2004;25(20):1776-1787. Doi: 10.1016/j.ehj.2004.07.010 25. Walker MD, Fleischer J, Rundek T, McMahon DJ, Homma S, Sacco R, et al. Carotid vascular abnormalities in primary hyperparathyroidism. J Clin Endocrinol Metab. 2009;94(10):38493856. Doi: 10.1210/jc.2009-1086

26. Ljunghall S, Palmer M, Akerstrom G, Wide L. Diabetes mellitus, glucose tolerance and insulin response to glucose in patients with primary hyperparathyroidism before and after parathyroidectomy. Eur J Clin Invest [Internet]. 1983;13(5):373-377. Doi: 10.1111/ j.1365-2362.1983.tb00116.x

27. Almqvist EG, Bondeson A-G, Bondeson L, Svensson J. Increased markers of inflammation and endothelial dysfunction in patients with mild primary hyperparathyroidism. Scand J Clin Lab Invest [Internet]. 2011;71(2):139-144.

28. Luigi P, Chiara FM, Laura Z, Cristiano M, Giuseppina C, Luciano C, et al. Arterial hypertension, metabolic syndrome and subclinical cardiovascular organ damage in patients with asymptomatic primaryhyperparathyroidism beforeand after parathyroidectomy: Preliminary results. Int J Endocrinol. 2012;2012:408295. Doi: 10.1155/2012/408295

29. Miyaki A, Maeda S, Yoshizawa M, Misono M, Sasai H, Shimojo N, et al. Is pentraxin 3 involved in obesity-induced decrease in arterial distensibility? J Atheroscler Thromb [Internet]. 2010;17(3):278284. 
30. Zanetti M, Bosutti A, Ferreira C, Vinci P, Biolo G, Fonda M, et al. Circulating pentraxin 3 levels are higher in metabolic syndrome with subclinical atherosclerosis: Evidence for association with atherogenic lipid profile. Clin Exp Med. 2009;9(3):243-248. Doi: 10.1007/s10238-009-0039-z

31. Osorio-Conles O, Guitart M, Chacón MR, Maymo-Masip E, Moreno-Navarrete JM, Montori-Grau M, et al. Plasma PTX3 protein levels inversely correlate with insulin secretion and obesity, whereas visceral adipose tissue PTX3 gene expression is increased in obesity. Am J Physiol Endocrinol Metab [Internet]. 2011;301(6):E1254-E1261. Doi: 10.1152/ajpendo.00163.2011

32. Miyaki A, Maeda S, Choi Y, Akazawa N, Eto M, Tanaka K, et al. Association of plasma pentraxin 3 with arterial stiffness in overweight and obese individuals. Am J Hypertens. 2013;26(10):1250-5. Doi: 10.1093/ajh/hpt103 